\title{
Armazenamento de metano à pressão reduzida em carvão ativado sob diferentes condições de temperatura e vazão de carga
}

\author{
Storage of methane at low pressure in activated \\ carbon under different temperature and \\ flow rate of charge conditions
}

Michael Feroldi ${ }^{1}$, Carlos Eduardo Borba ${ }^{2}$, Sidnei Dias Monteiro Júnior ${ }^{3}$, Helton José Alves ${ }^{1,3}$

\author{
${ }^{1}$ Programa de Pós Graduação em Engenharia de Energia na Agricultura, Universidade Estadual do Oeste do Paraná, Cas- \\ cavel, PR. \\ e-mail: michaelferoldi@gmail.com \\ ${ }^{2}$ Programa de Pós-graduação em Engenharia Química, Universidade Estadual do Oeste do Paraná, Toledo, PR. \\ e-mail: borba_deq@yahoo.com.br \\ ${ }^{3}$ Programa de Pós Graduação em Bioenergia, Universidade Federal do Paraná (UFPR-Setor Palotina), Palotina, PR \\ e-mail: jr10.monteiro@gmail.com; helquimica@gmail.com.
}

\begin{abstract}
RESUMO
O armazenamento de metano na forma adsorvida em carvão ativado (ANG - Adsorbed Natural Gas) tem despertado interesse nos últimos anos por empregar baixas pressões quando comparado ao gás natural comprimido (CNG - Compressed Natural Gas) e temperatura ambiente, e ainda por configurar uma alternativa importante à utilização de biometano oriundo da purificação de biogás em sistemas estacionários e veiculares. O presente estudo objetivou uma avaliação do armazenamento de metano em carvão ativado sob diferentes condições de temperatura e vazão de carga em um sistema protótipo. Metano foi inserido no sistema protótipo preenchido com carvão ativado vegetal a pressão reduzida (10 bar) utilizando planejamento fatorial $2^{2}$. Na dessorção do sistema foram levantadas cinéticas para identificar a melhor condição a ser adotada entre a condução dos ensaios de armazenamento para permitir o reuso do carvão ativado. O carvão ativado foi caracterizado com as técnicas de fisissorção de $\mathrm{N}_{2}$, para determinação das características texturais, picnometria de gás Hélio, para quantificação da densidade das partículas, e microscopia eletrônica de varredura para observação da estrutura morfológica. Nas condições adotadas no presente estudo, foi observado o aumento da capacidade de adsorção do carvão com a diminuição da temperatura e da vazão de metano no intervalo avaliado. Com o presente estudo foi possível aumentar a capacidade de armazenamento em aproximadamente 3 vezes (34,56 $\mathrm{V} \mathrm{V}^{-1}$ ) quando comparado com o cilindro vazio $\left(13,14 \mathrm{~V} \mathrm{~V}^{-1}\right)$, comprovando a eficácia do adsorvente e do conjunto de variáveis adotado. Em adição, o estudo cinético de dessorção de metano resultou na adoção da temperatura de $60{ }^{\circ} \mathrm{C}$ durante 30 min para total entrega do metano.
\end{abstract}

Palavras-chave: biometano, adsorção de gás, tecnologia ANG.

\begin{abstract}
Methane storage in adsorbed form in activated carbon (ANG - Adsorbed Natural Gas) has attracted attention in recent years by applying reduced pressures when compared to CNG (Compressed Natural Gas) technology and reduced temperatures, and also for representing an important alternative use of biomethane derived from biogas purification in vehicular and stationary systems. This study aimed an evaluation of methane storage in activated carbon under different conditions of temperature and flow rate of charge in a prototype system. Methane was inserted into the prototype system filled with activated vegetable carbon at reduced pressure (10 bar) using factorial design $2^{2}$. In the desorption of the system, kinetics was raised to identify the best condition to be adopted between the conduction of the storage trials to allow activated carbon reuse. Activated carbon was characterized with $\mathrm{N}_{2}$ physisorption for the determination of textural characteristics, Helium gas pycnometry for quantification of particle density, and scanning electron microscopy for observation of the morphological structure. Under the conditions adopted in this study, an increase was observed in activated
\end{abstract}


carbon adsorption capacity with decreasing temperature and flow rate during the evaluated range. With this study, it was possible to increase a cylinder storage capacity in approximately 3 times $\left(34,56 \mathrm{~V} \mathrm{~V}^{-1}\right)$ compared to the hollow cylinder $\left(13,14 \mathrm{~V} \mathrm{~V}^{-1}\right)$, proving the effectiveness of the adsorbent and the set of variables adopted. In addition, the kinetic study of methane desorption resulted in the adoption of $60{ }^{\circ} \mathrm{C}$ for 30 min for the complete methane delivery.

Keywords: biomethane, gas adsorption, ANG technology.

\section{INTRODUÇÃO}

A utilização da biomassa moderna, em virtude da preocupação para com o meio ambiente e a segurança energética, é fruto da transição do uso de fontes fósseis de energia para as fontes renováveis, observada nos últimos anos. Por sua vez, tal transição tem gerado significativa atenção a diversos tipos de biocombustíveis, denominados combustíveis de origem renovável, tendo o biogás, que é obtido de diversas matérias-primas (resíduos ou não), como um dos principais representantes com possibilidade de ser utilizado diretamente ou em motogeradores de energia elétrica [1].

As aplicações do biogás são diversas e estas são na maioria das vezes dependentes de sua composição química, a qual é responsável pelas suas características energéticas. O poder calorífico do biogás é o fator chave para utilização deste na conversão em energia e, devido a isto, diversas técnicas de purificação são empregadas para o enriquecimento em metano, utilizadas principalmente para a remoção de $\mathrm{CO}_{2}$ e $\mathrm{H}_{2} \mathrm{~S}$ [2].

Uma vez que as principais aplicações energéticas do biogás estão ligadas ao alto conteúdo em metano, responsável pelo poder calorífico, e a ausência de compostos corrosivos e abafantes $\left(\mathrm{H}_{2} \mathrm{~S}\right.$ e $\left.\mathrm{CO}_{2}\right)$, o biogás purificado, ou biometano como é denominado pela Resolução 8/2015 da ANP (Agência Nacional do Petróleo, Gás Natural e Biocombustíveis), pode ser facilmente aplicado como substituinte ou juntamente ao gás natural em veículos automotores devido à semelhança físico-química e miscibilidade [3].

Devido ao grande potencial e perspectivas no setor, a utilização de biometano no setor veicular tem motivado o estudo e aprimoramento das tecnologias envolvidas no transporte e armazenamento de combustíveis gasosos, o que é extremamente importante para o desenvolvimento do setor [4,5]. Desta forma, dentre as tecnologias utilizadas para o transporte e armazenamento de gás natural/biometano, a que emprega o fenômeno de adsorção em sólidos adsorventes porosos (ANG - Adsorbed Natural Gas) tem despertado grande interesse por apresentar-se como alternativa às tecnologias que envolvem altas quantidades de energia em seus processos de compressão e liquefação de gases [6].

Dentre os inúmeros materiais sólidos adsorventes utilizados no armazenamento de gás natural/metano/biometano, o carvão ativado é o mais utilizado no setor, devido à facilidade de obtê-los e a infinidade de precursores, também muitas vezes de fácil acesso [7]. Carvões ativados são materiais sólidos que possuem estrutura bastante porosa e alta variabilidade no diâmetro destes poros, sendo estes microporosos (< $2 \mathrm{~nm}$ ), mesoporosos (2-50 nm) e macroporosos (> $50 \mathrm{~nm})$. Sua área específica também é uma característica bastante variável que depende do processo de ativação (física e/ou química), podendo alcançar de 500 a 3000 $\mathrm{m}^{2} \mathrm{~g}^{-1}[8]$.

O uso de ferramentas estatísticas para prever ou otimizar processos é fundamental frente a enormidade de combinações de fatores variáveis, portanto planejamentos experimentais do tipo fatorial combinados com ferramentas de resposta de dados como ANOVA, superfícies de resposta e gráfico de Pareto são necessários para montar planejamentos experimentais com o menor número de experimentos e para compreender os resultados combinados e ainda prever outras possíveis combinações para alcançar melhores rendimentos [9].

Em virtude da escassez de estudos que envolvem pressões reduzidas (10 bar) em um sistema de escala de aplicação real no estudo do armazenamento de metano na forma adsorvida, o presente trabalho tem por objetivo avaliar o armazenamento de metano em carvão ativado sob diferentes condições de temperatura e vazão de carga de metano em um sistema de adsorção protótipo com auxílio de ferramentas estatísticas para planejamento e compreensão dos resultados.

\section{MATERIAIS E MÉTODOS}

Para realização dos testes de armazenamento de metano foram utilizadas $190 \mathrm{~g}$ de carvão ativado comercial com diâmetro de 2 mm (8x30) proveniente de cascas de babaçu da Brascarbo Agroindustrial Ltda. O material foi seco em estufa a $100{ }^{\circ} \mathrm{C}$ por $24 \mathrm{~h}$ antes dos ensaios de armazenamento para evitar a presença de umidade. 


\subsection{Caracterização}

\subsubsection{Fisissorção de $\mathrm{N}_{2}$}

Foram obtidas isotermas de adsorção/dessorção de $\mathrm{N}_{2}$ à $77 \mathrm{~K}$ com auxílio do equipamento Nova 2000e da Quantachrome, tendo as amostras sido submetidas a pré-tratamento a $200{ }^{\circ} \mathrm{C}$ por $4 \mathrm{~h}$, sob vácuo a fim de retirar toda umidade e espécies orgânicas eventualmente adsorvidas na superfície do material. As áreas específicas foram determinadas com auxílio do método de Brunauer-Emmet-Teller (BET) (p/p0 = 0,05-0,30), os volumes de poros calculados à pressão relativa de 0,99 e os volumes de microporos foram estimados de acordo com o método de Dubinin-Radushkevich (DR). O diâmetro médio de poros foi determinado a partir do método BJH (Barret-Joyer-Halenda) [10, 11].

\subsubsection{Microscopia eletrônica de varredura}

A microscopia eletrônica de varredura foi empregada com o intuito de determinar a morfologia e o tamanho médio das partículas. Nesta análise foi utilizado um microscópio eletrônico de varredura (VEGA3 TESCAN), onde as amostras, em forma de pó, foram espalhadas no porta amostra sobre uma fita de carbono dupla face, sendo posteriormente secas e metalizadas com uma fina camada de ouro na superfície ("sputtering”). As micrografias foram obtidas em diversos aumentos em módulo BSE (backscattering) com detectores de elétrons retroespalhados e módulo SE com detectores de elétrons retroespalhados e secundários.

\subsubsection{Picnometria de gás Hélio}

A picnometria de gás Hélio foi realizada nos carvões ativados por meio do equipamento Quantachrome Ultrapycnometer 1000, a fim de possibilitar a determinação do volume verdadeiro dos adsorventes, descontando os espaços vazios dos poros, conforme descrito por Alves et al. [12] na Equação 1:

$$
V s=V a-\frac{V e}{\frac{P 1}{P 2}-1}
$$

na qual P1 é a pressão inicial e P2 a final (P1 > P2), Va o volume da câmara da amostra, Ve o volume da câmara de expansão e Vs o volume do sólido.

\subsection{Ensaios de armazenamento de metano}

Para condução dos ensaios no Laboratório de Catálise e Produção de Biocombustíveis (LabCatProBio) da Universidade Federal do Paraná, Setor Palotina, utilizou-se metano $\left(\mathrm{CH}_{4}\right)($ White Martins, pureza = 99,5\%) para injeção no sistema protótipo de armazenamento, constituído de um cilindro de adsorção (0,465 L) com acomodação para carga de adsorvente, bomba compressora de baixa vazão ajustável e forno de dessorção. Os ensaios de armazenamento foram conduzidos à pressão máxima de 10 bar empregando planejamento fatorial $2^{2}$ com triplicata no ponto central, no qual variou-se a temperatura de adsorção e a velocidade de injeção de metano, conforme descrito na Tabela 1. Foi utilizado o software Statistica 8.0 para elaboração de superfícies de resposta e gráficos de Pareto para avaliação dos resultados estatísticos.

Tabela 1: Planejamento fatorial $2^{2}$ para os ensaios de adsorção

\begin{tabular}{ccccc}
\hline \multirow{2}{*}{ ENSAIO } & \multicolumn{2}{c}{ TEMPERATURA } & \multicolumn{2}{c}{ VAZÃO } \\
\cline { 2 - 5 } & CODIFICADO & ${ }^{\circ} \mathbf{C}$ & CODIFICADO & $\mathbf{~ m L ~ s}^{-1}$ \\
\hline $\mathbf{1}$ & - & 20 & - & 3,65 \\
$\mathbf{2}$ & + & 40 & - & 3,65 \\
$\mathbf{3}$ & - & 20 & + & 7,3 \\
$\mathbf{4}$ & + & 40 & + & 7,3 \\
$\mathbf{5}$ & PC & 30 & PC & 5,54 \\
$\mathbf{6}$ & PC & 30 & PC & 5,54 \\
$\mathbf{7}$ & PC & 30 & PC & 5,54 \\
\hline
\end{tabular}

PC: ponto central

Após atingida a pressão de interesse (10 bar), a quantidade de gás armazenado foi obtida por pesagem 
do sistema, registrando a diferença de massa posterior à adsorção e anterior à adsorção de $\mathrm{CH}_{4}$ e em seguida foi esvaziado. Os resultados mássicos foram transformados em base volumétrica nas condições padrão (298 K e 1 atm), de acordo com a Equação 2:

$$
V_{C H 4}=\frac{n * R * T_{0}}{P_{0}}
$$

Finalmente o sistema foi submetido à temperatura de $60^{\circ} \mathrm{C}$ (investigada na seção 3.3) para total remoção das moléculas de metano adsorvidas nos poros dos adsorventes, regenerando o material para os próximos ciclos de adsorção.

\section{RESULTADOS E DISCUSSÃO}

\subsection{Caracterização}

Na Tabela 2 estão descritas as propriedades texturais obtidas por fisissorção de $\mathrm{N}_{2}$ do carvão ativado utilizado nos ensaios de adsorção de $\mathrm{CH}_{4}$.

Tabela 2: Propriedades texturais do carvão ativado obtidas por fisissorção de $\mathrm{N}_{2}$

\begin{tabular}{lcccc}
\cline { 2 - 5 } & $\begin{array}{c}\text { ÁREA } \\
\text { ESPECÍFICA } \\
\left(\mathbf{m}^{\mathbf{2}} \mathbf{g}^{-1}\right)\end{array}$ & $\begin{array}{c}\text { VOLUME } \\
\text { TOTAL DE } \\
\text { POROS } \\
\left(\mathbf{c m}^{\mathbf{3}} \mathbf{g}^{-1}\right)\end{array}$ & $\begin{array}{c}\text { VOLUME DE } \\
\text { MICROPOROS } \\
\left(\mathbf{c m}^{\mathbf{3}} \mathbf{g}^{-1}\right)\end{array}$ & $\begin{array}{c}\text { DIÂMETRO } \\
\text { MÉDIO } \\
(\AA)\end{array}$ \\
\hline CARVÃO ATIVADO & 603,22 & 0,3278 & 0,3152 & 35,4 \\
\hline
\end{tabular}

A partir destes resultados, foi possível determinar que o carvão ativado testado no presente estudo possui elevada área específica além de elevado volume de microporos, muito semelhantes aos obtidos por Arami-Niya et al. [10] com cascas de palma que apresentaram área específica de $615 \mathrm{~m}^{2} \mathrm{~g}^{-1}$ e volume total e de microporos de 0,28 e $0,26 \mathrm{~cm}^{3} \mathrm{~g}^{-1}$, respectivamente. Tais resultados reservam características favoráveis à adsorção de $\mathrm{CH}_{4}$, além de tratar-se de um adsorvente de baixo custo e comercialmente difundido.

Para melhor identificação das características texturais e de comportamento do carvão ativado, foi construída e apresentada na Figura 1 a respectiva isoterma de adsorção de $\mathrm{N}_{2}$.

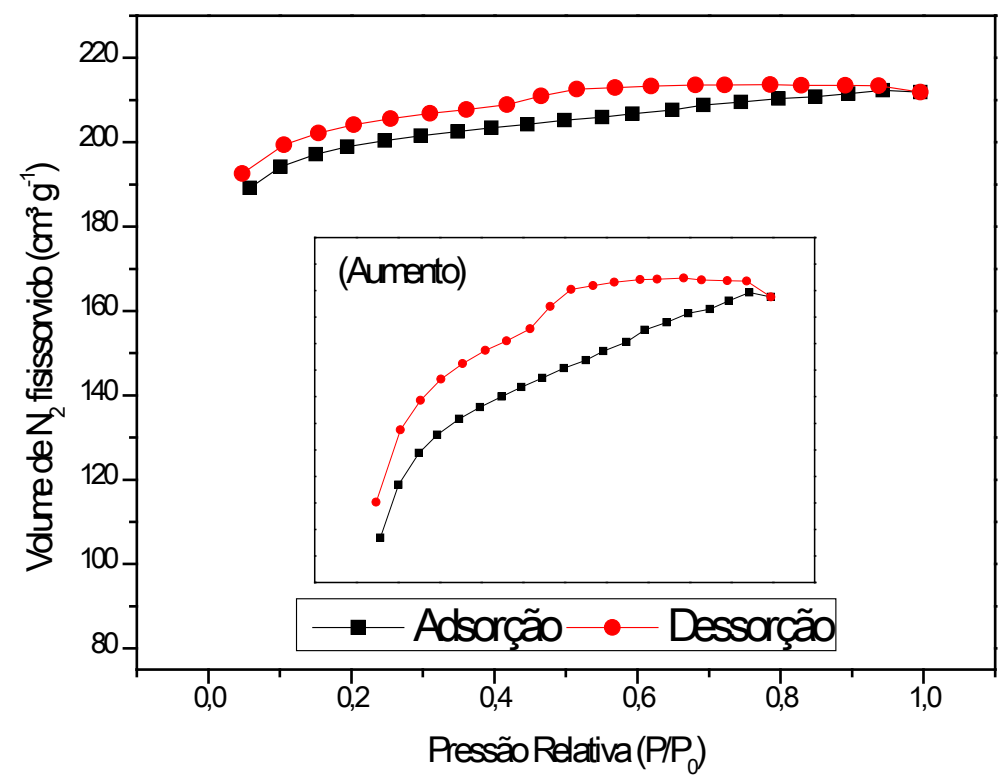

Figura 1: Isoterma de adsorção/dessorção de $\mathrm{N}_{2}$ a $77 \mathrm{~K}$ no carvão ativado.

A isoterma exposta na parte superior do gráfico (obedecendo a escala) pode ser classificada como do tipo Ib, característica de sólidos microporosos largos com possível presença de mesoporos estreitos, confor- 
me classificação IUPAC (International Union of Pure and Applied Chemistry) [13]. A partir de um aumento de escala na isoterma é possível observar a presença de histereses provenientes do processo de condensação capilar, possivelmente devido à grande variação no tamanho e morfologia dos poros como consequência das matérias-primas utilizadas na fabricação do carvão, bem como do tratamento realizado durante sua ativação e do tipo de grânulos formados. As histereses observadas são do tipo H4, indicando também a presença de mesoporos.

A partir da Figura 2 é possível verificar as características morfológicas do carvão ativado por meio de micrografias obtidas em microscópio eletrônico de varredura (MEV).

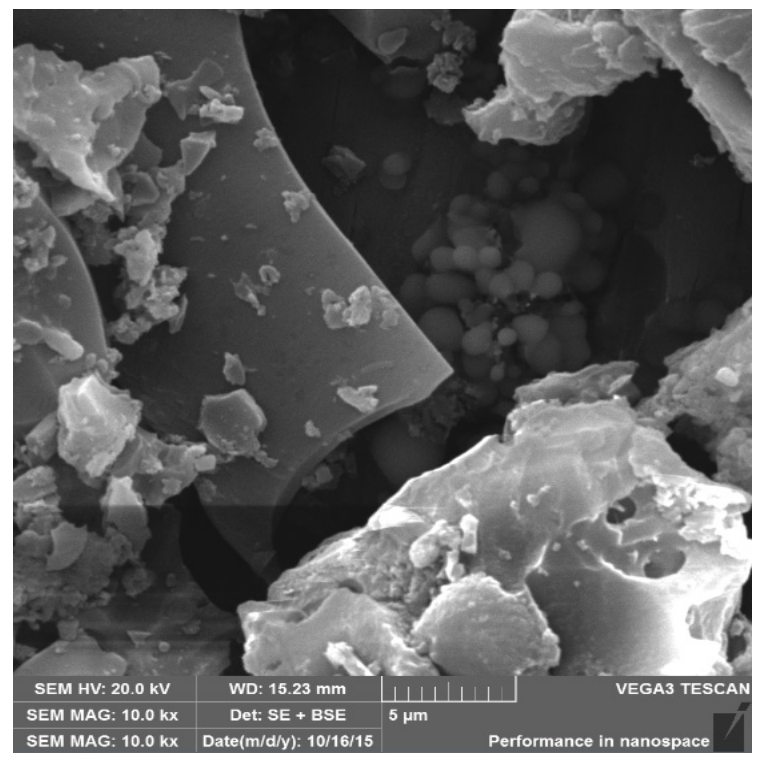

Figura 2: Micrografia do carvão ativado em aumento de 10000 vezes.

Observando a Figura 2 é possível notar que o carvão ativado apresenta alta heterogeneidade morfológica com partículas de tamanhos e formatos irregulares com superfícies angulares e esféricas variando de 1 $\mu \mathrm{m}$ a $20 \mu \mathrm{m}$.

A picnometria de gás Hélio permitiu a determinação do volume absoluto das partículas sólidas do carvão ativado, livre da contabilização dos poros [7,14] e este valor $(2,704 \pm 0,001)$ apresentou-se ligeiramente superior ao obtido por Policicchio et al. [7], e proporcionou porosidade de leito de aproximadamente 84,9\%, muito próximo ao obtido por Rios et al. [15].

\subsection{Armazenamento de $\mathrm{CH}_{4}$ na forma adsorvida}

Os resultados dos testes de adsorção provenientes do planejamento fatorial $2^{2}$ do presente estudo, expostos na Tabela 3 juntamente com o ensaio do cilindro sem adsorvente, foram obtidos a partir da conversão das capacidades mássicas medidas para os respectivos volumes de $\mathrm{CH}_{4}$ adsorvido por grama de adsorvente ( $\mathrm{L} \mathrm{g}^{-1}$ ) e por volume de cilindro $\left(\mathrm{V} \mathrm{V}^{-1}\right)$.

Tabela 3: Armazenamento de $\mathrm{CH}_{4}$ em volume por grama de adsorvente $\left(\mathrm{L} \mathrm{g}^{-1}\right)$ e por volume de cilindro $\left(\mathrm{V} \mathrm{V}^{-1}\right)$

\begin{tabular}{|c|c|c|c|}
\hline \multicolumn{2}{|c|}{ CILINDRO VAZIO } & & \\
\hline & TEMP. I VAZÃO & $\mathrm{L} \mathrm{g}^{-1}$ & $\mathrm{VV}^{-1}$ \\
\hline 1 & -- & 0,0846 & 34,56 \\
\hline 2 & +- & 0,0723 & 29,57 \\
\hline 3 & -+ & 0,0804 & 32,84 \\
\hline 4 & ++ & 0,0643 & 26,28 \\
\hline 5 & PC & 0,0747 & 30,52 \\
\hline 6 & PC & 0,0776 & 31,70 \\
\hline 7 & PC & 0,0743 & 30,34 \\
\hline
\end{tabular}

PC: Ponto Central 
Conforme observado na Tabela 3, o planejamento fatorial proporcionou alta variabilidade (positiva) de resultados entre os ensaios de armazenamento, e o ensaio \#1 (20 ${ }^{\circ} \mathrm{C}$ e 3,65 $\left.\mathrm{mL} \mathrm{s}^{-1}\right)$ foi responsável pela maior capacidade de armazenamento de $\mathrm{CH}_{4}\left(34,56 \mathrm{~V} \mathrm{~V}^{-1}\right)$ dentre todas as combinações analisadas no planejamento experimental. Este resultado confrontado com o volume útil do cilindro (0,465 L) possibilitou o armazenamento de 16,07 $\mathrm{L}$ de $\mathrm{CH}_{4}$, comprovando que o emprego de adsorventes no armazenamento de $\mathrm{CH}_{4}$ é eficaz, aumentando aproximadamente 3 vezes nas condições utilizadas no presente estudo quando comparado ao cilindro sem adsorvente $\left(13,14 \mathrm{~V} \mathrm{~V}^{-1}\right)$.

$\mathrm{O}$ desempenho do estudo de armazenamento de $\mathrm{CH}_{4}$ ainda foi investigado com auxílio de ferramentas estatísticas a fim de determinar a importância de cada variável testada (temperatura e vazão). Para tal, foram geradas superfícies de resposta e gráficos de Pareto, expostos nas Figuras 3 e 4, respectivamente.

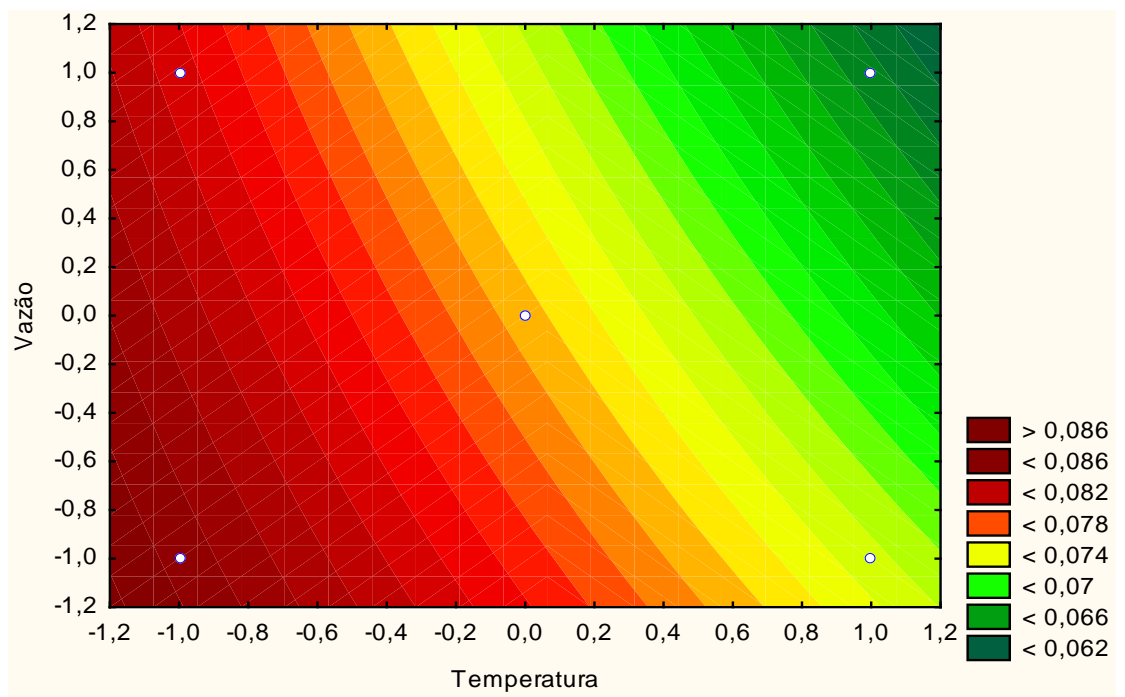

Figura 3: Superfície de resposta dos ensaios de armazenamento de $\mathrm{CH}_{4} \mathrm{em} \mathrm{L} \mathrm{g}^{-1}$.

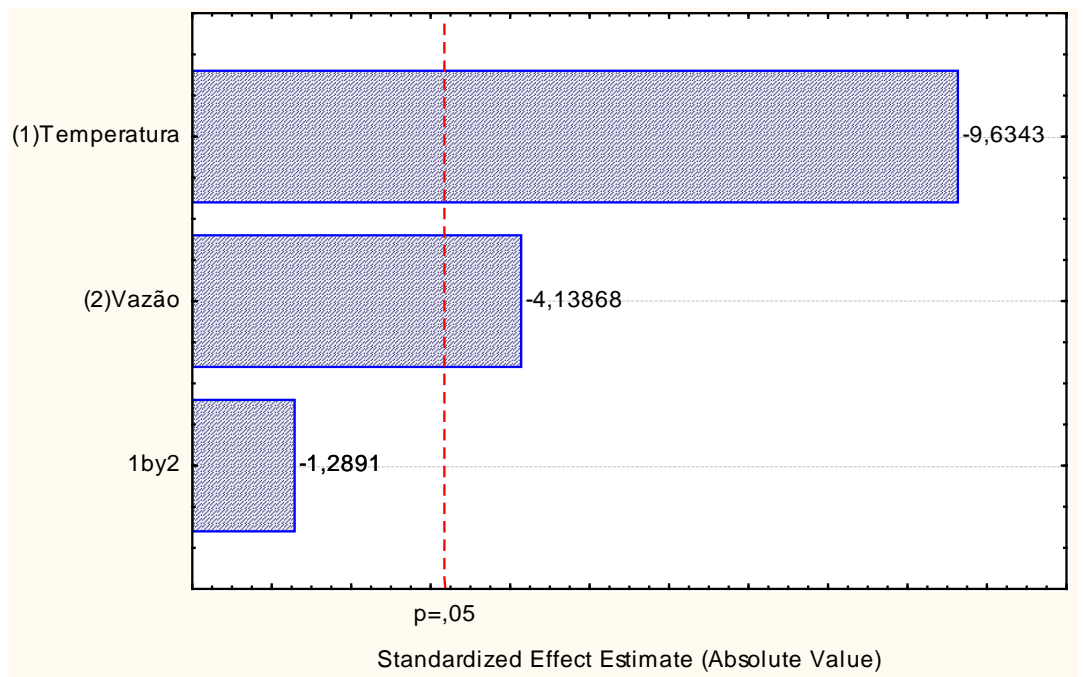

Figura 4: Gráfico de Pareto dos ensaios de armazenamento de $\mathrm{CH}_{4}$.

Como pode ser observado na Figura 3, os melhores resultados de adsorção de $\mathrm{CH}_{4}$ estão localizados próximos aos pontos de menor vazão e temperatura, o que corroboram com o gráfico de Pareto (Figura 4) que plota o nível de significância de cada variável. Observa-se que os coeficientes estatísticos possuem valores negativos, o que indicam que a melhor resposta tende aos ensaios com as menores temperaturas e vazões. Os níveis de significância das variáveis apresentaram valor de " $p$ ” (0,002374 para temperatura e 0,025609 para vazão) abaixo de 0,05 (máximo para significância) e por isto representaram significância, o contrário da interação entre as duas variáveis que apresentou valor de 0,287767 (portanto acima de 0,05). Assim, estas 
análises estatísticas permitiram a identificação da significância de ambas as variáveis testadas (temperatura e vazão) configurando melhor significância para a temperatura, uma vez que o fenômeno de adsorção de gases é exotérmico [16].

\subsection{Cinética de Dessorção}

A investigação do processo de dessorção de $\mathrm{CH}_{4}$ foi desenvolvida a fim de identificar a condição térmica mais branda necessária ao total esvaziamento das partículas do carvão ativado para novos reciclos de adsorção de $\mathrm{CH}_{4}$. Este estudo pode ser observado na Figura 5 para melhor compreensão.

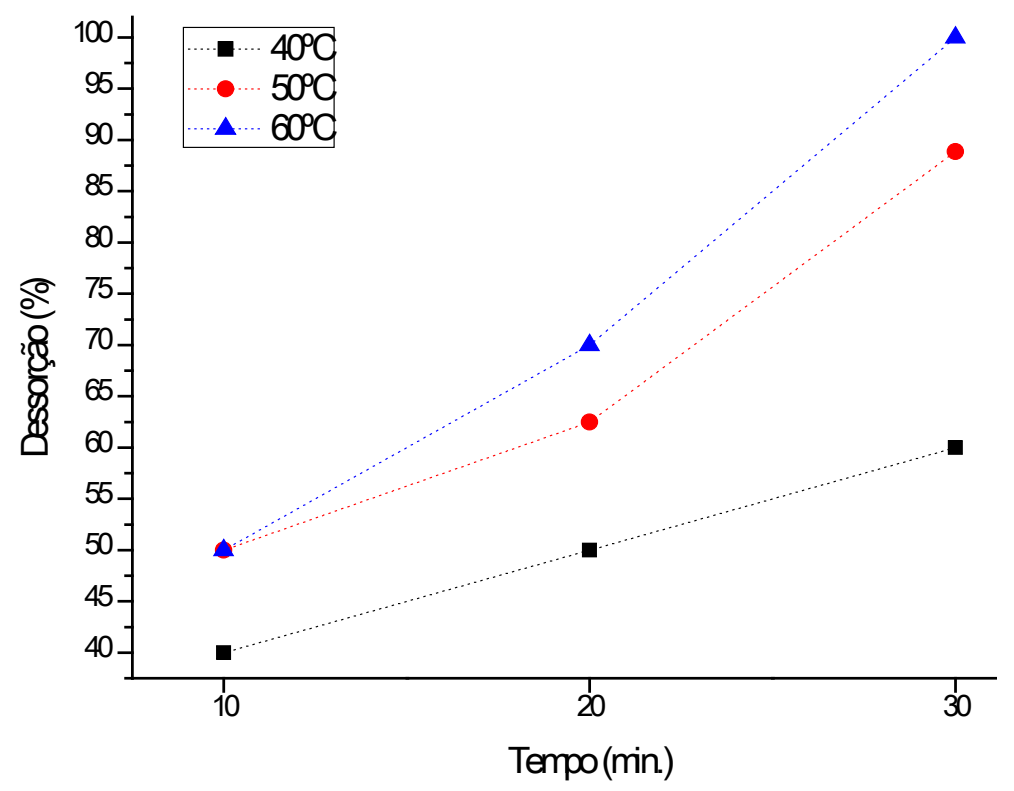

Figura 5: Estudo cinético de dessorção de $\mathrm{CH}_{4}$ para recuperação do carvão ativado.

A partir do estudo cinético foi possível determinar que a temperatura de $60{ }^{\circ} \mathrm{C}$ durante 30 min é responsável pela quase completa dessorção de $\mathrm{CH}_{4}$ dos poros do carvão ativado empregado após as condições de adsorção empregadas no presente estudo, não devendo ser generalizadas para outras configurações sem estudo prévio.

\section{CONCLUSÕES}

O presente estudo proporcionou resultados interessantes a partir de variáveis pouco exploradas na literatura pesquisada. O emprego de um sistema equipado com temperatura e vazão ajustáveis no armazenamento de $\mathrm{CH}_{4}$ combinado com forno de dessorção foi fundamental na investigação do comportamento do carvão ativado, visando condições próximas à realidade de aplicações. Nas condições empregadas $\left(10\right.$ bar, $20^{\circ} \mathrm{C}$ e 3,65 $\mathrm{mL} \mathrm{s}^{-1}$ ), foi possível alcançar a capacidade de $34,56 \mathrm{~V} \mathrm{~V}^{-1}$ no armazenamento de $\mathrm{CH}_{4}$, projetando o presente estudo para aplicações voltadas aos setores rural, com o uso de sistemas de estocagem de biometano em períodos de produção intermitente, e veicular.

\section{AGRADECIMENTOS}

Os autores agradecem ao Cibiogás, à Funarbe e à Capes, pelo financiamento do projeto e de bolsa.

\section{BIBLIOGRAFIA}

[1] COIMBRA-ARAÚJO, C.H., MARIANE, L., BLEY JR, C., et al., "Brazilian case study for biogas energy: Production of electric power, heat and automotive energy in condominiums of agroenergy", Renewable and Sustainable Energy Reviews, v. 40, pp. 826-839, 2014.

[2] CHMIELEWSKI, A.G., URBANIAK, A., WAWRYNIUK, K., "Membrane enrichment of biogas from two-stage pilot plant using agricultural waste as a substrate”, Biomass and Bioenergy, v. 58, pp. 219-228, 2013.

[3] XIAO, Y., YUAN, H., PANG, Y., et al., "CO2 Removal from Biogas by Water Washing System”, Chinese Journal of Chemical Engineering, v. 22, n. 8, pp. 950-953, 2014. 
[4] BORDELANNE, O., MONTERO, M., BRAVIN, F., et al., "Biomethane CNG hybrid: A reduction by more than $80 \%$ of the greenhouse gases emissions compared to gasoline”, Journal of Gas Science and Engineering, v. 3, pp. 617-624, 2011.

[5] MURPHY, J.D., BROWNE, J., ALLEN, E., et al., "The resource of biomethane, produced via biological, thermal and electrical routes, as a transport biofuel”, Renewable Energy, v. 55, pp. 474-479, 2013.

[6] FEROLDI, M., NEVES, A.C., BACH, V.R., et al., "Adsorption technology for the storage of natural gas and biomethane from biogas”, International Journal of Energy Research, v. 40, n. 14, pp. 1890-1900, 2016.

[7] POLICICCHIO, A., MACCALLINI, E., AGOSTINO, R.G., et al., "Higher methane storage at low pressure and room temperature in new easily scalable large-scale production activated carbon for static and vehicular applications”, Fuel, v. 104, pp. 813-821, 2013.

[8] OUBAGARANADIN, J.U.K., MURTHY, Z.V.P., Activated carbons: Classifications, properties and applications, In: James F. Kwiatkowski (Ed.), Activated Carbon: Classifications, Properties and Applications. Nova Science Publishers Inc, USA, pp. 239-266, 2011.

[9] REGTI, A., LAAMARI, M.R., STIRIBA, S.E., et al., "Use of response factorial design for process optimization of basic dye adsorption onto activated carbon derived from Persea species", Microchemical Journal, v. 130, pp. 129-136, 2017.

[10] ARAMI-NIYA, A., DAUD, W.M.A.W., MJALLI, F.S., "Comparative study of the textural characteristics of oil palm shell activated carbon produced by chemical and physical activation for methane adsorption", Chemical Engineering Research and Design, v. 89, pp. 657-664, 2011.

[11] ROUQUEROL, F., ROUQUEROL, J., SING, K. Adsorption by Powders \& Porous Solids: Principles, Methodology and Applications. Academic, London (1999).

[12] ALVES, H.J., FREITAS, M.R., MELCHIADES, F.G., et al., "Dependence of surface porosity on the polishing depth of porcelain stoneware tiles”, Journal of the European Ceramic Society, v. 31, pp. 665-671, 2011.

[13] THOMMES, M., KANEKO, K., NEIMARK, A.V., et al., "Physisorption of gases, with special reference to the evaluation of surface area and pore size distribution (IUPAC Technical Report)", Pure and Applied Chemistry, v. 87, pp. 1051-1069, 2015.

[14] MINUTO, F.D., POLICICCHIO, A., ALOISE, A., et al., "Liquid-like hydrogen in the micropores of commercial activated carbons”, International Journal of Hydrogen Energy, v. 40, pp. 14562-14572, 2015.

[15] RIOS, R.B., BASTOS-NETO, M., AMORA JR, M.R., et al., "Experimental analysis of the efficiency on charge/discharge cycles in natural gas storage by adsorption”, Fuel, v. 90, pp. 113-119, 2011.

[16] MENON, V.C., KOMARNENI, S. "Porous Adsorbents for Vehicular Natural Gas Storage: A Review", Journal of Porous Materials, v. 5, pp. 43-58, 1998. 